\title{
Full volumetric reconstruction optical coherence tomography utilizing compressive sensing
}

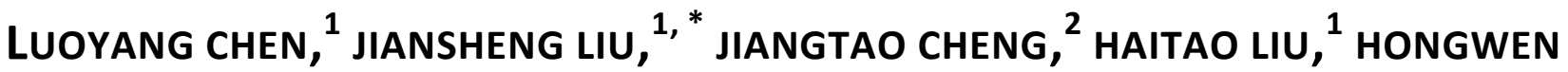 \\ $\mathrm{ZHOU}^{1}$ \\ ${ }^{1}$ Shool of Electronic and Information Engineering, Beihang University, 37 Xueyuan Rd,Beijing 100191,China \\ ${ }^{2}$ Department of Mechanical Engineering Virginia Tech,635 Prices Fork Road, Blacksburg, VA 24061, USA \\ *Corresponding author: jsliu@buaa.edu.cn
}

Received XX Month XXXX; revised XX Month, XXXX; accepted XX Month XXXX; posted XX Month XXXX (Doc. ID XXXXX); published XX Month XXXX

3D optical coherence tomography imaging (OCT) combined with compressive sensing (CS) has been proved to be an attractive and effective tool in a variety of fields, such as medicine and biology. To achieve high quality imaging while using as less CS sampling rate as possible is the goal of this approach. Here we present an innovative single step fully 3D CS-OCT volumetric image recovery method, in which 3D OCT volumetric image of the object is compressively sampled via our proposed CS coding strategies in all three dimensions while its sparsity is simultaneously taken into consideration in every direction. The object can be directly recovered as the whole volume reconstruction via our advanced full 3D CS reconstruction algorithm. The numerical simulations of a human retina OCT volumetric image reconstruction by our method demonstrate a PSNR of as high as $38 \mathrm{~dB}$ at a sampling rate of less than $10 \%$.

OCIS codes: (170.4500) Image Optical coherence tomography; (100.30100) Image reconstruction techniques; (110.2990) Image formation theory;

\section{Introduction}

During the past few years, 3D OCT volumetric image, which can provide an ultra-high resolution and volumetric visualization image, has been considered as a powerful and effective tool in many application fields, such as medicine and life science ${ }^{1-7}$. However, this process is highly time-consuming8-10 due to a large number of datapoints in each acquisition is required. Moreover, it is susceptible to some unavoidable motion artifact that results in degradation and even deterioration ${ }^{11-13}$ of the image resolution.

In order to address the limitations of the conventional 3D OCT imaging method, compressive sensing (CS) techniques-based OCT systems (CSOCT) have attracted significant attention during the last few years ${ }^{14-16}$. CS is an amazing signal processing theory where a signal can be recovered with a much less number of non-adaptive linear measurements than that required by Shannon/Nyquist sampling theory ${ }^{17}$. In this respect, CS-OCT can provide a dramatic reduction in the number of sampling measurements with less loss of reconstruction information ${ }^{18-20}$. Various 3D CS-OCT methods have been reported for the high-speed and high-resolution OCT applications ${ }^{14,15,21}$, in which either A-scan or B-scan is CS sampled without considering the sparsity or correlation with other scan directions and 3D OCT images was reconstructed by these CS sampled scan data on CS reconstruction theory. Nevertheless, such a method would not be expected to reduce redundant measurements for the whole 3D OCT image since only 1D or 2D images data were CS sampled and reconstructed, meanwhile most of natural 3D OCT images exhibit sparse characteristics in each dimension of 3D. Lately, a full 3D CS-OCT method sampling and recovering in all three directions to further reduce the number of CS sampling acquisitions with low image quality degradation, was described by Xu et $\mathrm{al}^{22}$. The CS sampling of a 3D OCT image in the Cscan direction was implemented by omitting one or more whole slices of B-scans obtained by selecting and CS sampling some A-scan lines while abandoning rest of unselected whole lines, and a three-step CS strategy were adopted for the reconstruction of the 3D OCT image as shown in Fig. 1. A high resolution vivo biological tissue's 3D OCT image was reconstructed from only about $20 \%$ measurements of the original OCT volumetric image.

In this paper, a novel single step fully 3D CS-OCT volumetric image reconstruction approach is described. In this approach, B-scans are CS sampled while the CS sampling pattern of each B-scan is determined by both the sparsity of the scan itself and the correlations with its top and bottom neighboring B-scans as depicted in Fig. 2. In this way, the objective 3D OCT volumetric image is CS sampled simultaneously in all three dimensions, and the total number of measurements can be dramatically reduced. Then, the measurement data was used to recover the OCT volumetric image via our full 3D CS-OCT volumetric reconstruction algorithm (see Method section below). Therefore, it is simply but very effective to reconstruct the high-resolution 3D OCT volumetric image from small amounts of measurements data for timesaving purpose.

\section{Methods}

To better understand the single step fully 3D CS-OCT approach, a sketch of the process is presents in Fig.1. A 3D OCT image 
$X \in \mathbb{R}^{n_{1} \times n_{2} \times n_{3}}$, where $\mathrm{n}_{1}, \mathrm{n}_{2}, \mathrm{n}_{3}$ represent the number of pixels in each dimensional of the 3D OCT image, is vectorized into a 1D signal $x \in \mathbb{R}^{n \times 1}$ of a length of $\mathrm{n} \triangleq\left(\mathrm{n}_{1}, \mathrm{n}_{2}, \mathrm{n}_{3}\right)$; and then the measurement vector $y \in \mathbb{R}^{m \times 1}$ is obtained by computing the inner products between a measurement matrix $A \in \mathbb{R}^{m \times n}(\mathrm{~m}<<\mathrm{n})$ and the vectorized 3D CS-OCT volume image $X$. The incoherent sampling 3D CS-OCT volume image process can be written as

$$
y=A x+\eta
$$

where $\eta \in \mathbb{R}^{m \times 1}$ is the measurement noise vector. Importantly, the measurement or sensing matrix $A \in \mathbb{R}^{m \times n}$ must be incoherent with the sparse basis of the 3D OCT volume image and satisfy the Restricted Isometry Property (RIP) criterion according to CS theory 23,24 . A number of sensing matrices meet both two requirements, including random Gaussian matrices, Fourier transform matrices, and WalshHadamard matrices.

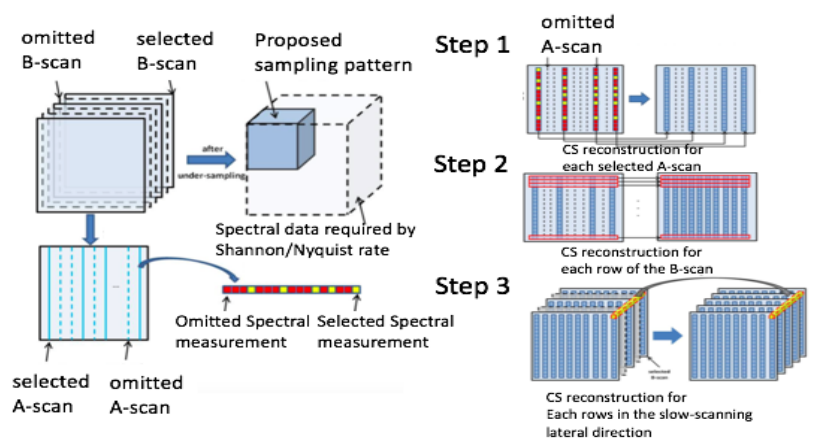

Fig. 1. Three step full 3D CS-OCT volume reconstruction scheme22.

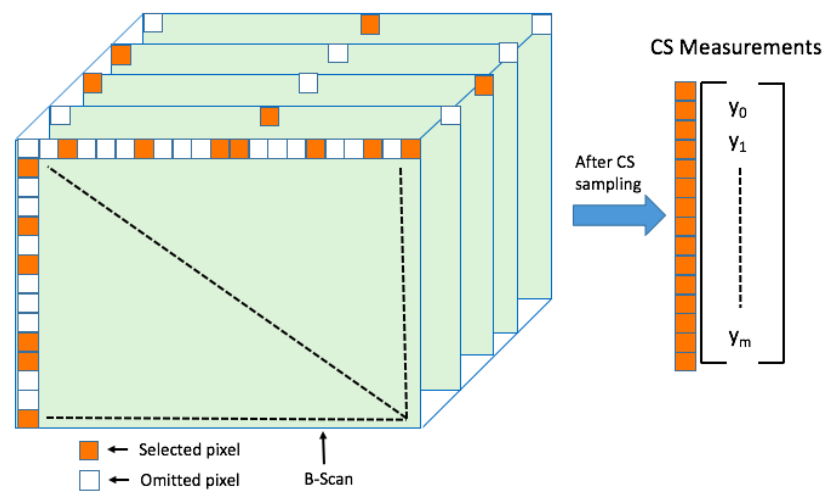

Fig. 2. Single step protocol-based fully 3D CS-OCT volume sampling pattern scheme.

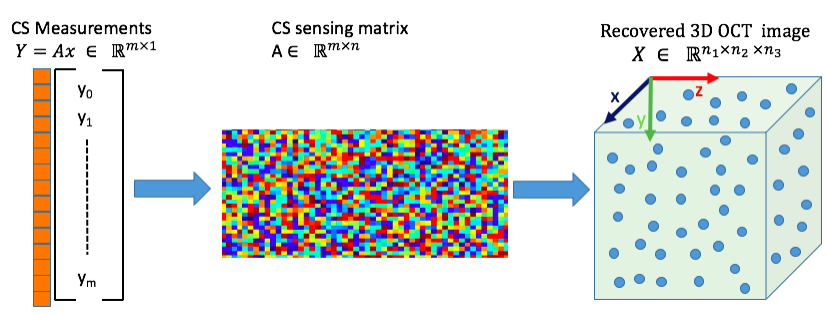

Fig. 3. Single step protocol-based full 3D CS-OCT volumetric reconstruction scheme.

The goal of this work is to recover the whole correct 3D OCT volumetric image $X \in \mathbb{R}^{n_{1} \times n_{2} \times n_{3}}$ from the underdetermined CS- based encoding system that given the measurement vector $y \in \mathbb{R}^{m \times 1}$ and sensing matrix $A \in \mathbb{R}^{m \times n}$ as depicted in Fig. 3. Let assume the top-left-back corner of the 3D CS-OCT volumetric image is the origin, and the pixel position in the 3D CS-OCT volumetric image is denoted by $I=\left(\dot{t}_{1}, \dot{t}_{2}, \dot{t}_{3}\right)$, where $\dot{t}_{1}, \dot{t}_{2}, \dot{t}_{3}$ are horizontal (B-scan) and vertical (Ascan) offsets the origin, and the slice number(C-scan), respectively. Thus the pixel values $P \in \mathbb{R}^{n_{1} \times n_{2} \times n_{3}}$, where $P_{I}$ is the pixel value at a position I $\in \mathbb{R}^{n_{1} \times n_{2} \times n_{3}}$, can be recovered from $y$ by solving the constrained minimization problem

$$
\underset{x}{\operatorname{argmin}} \varphi(x) \text { s.t. } A x=y
$$

where $\varphi(\mathrm{x})$ is a regularization term, such as $l_{0}$-norm,$l_{1}$-norm, and $l_{2}$-norm etc. Typically, real-world signals are not exactly sparse in our familiar space-time coordinates, but they can be accurate approximations in a proper orthogonal basis or linear transformation, such as Wavelet, Fourier transform, discrete cosine transformation, etc. In this way, the regularization term in Eq. (2) can be rewritten as

$$
\varphi(x)=\|L x\|_{p}
$$

where $L$ is a linear transformation operator which yields a sparse representation of $x$, and $\|.\|_{p}$ denotes the p-norm. In general, $l_{0}$-norm regularization can be recovered from a K-sparse signal exactly25; however, it is a NP-hard problem and hard to solve. Since $l_{2}$-norm measures signal energy rather than signal sparsity and $l_{1}$-norm minimization can yield nearly the same result as the $l_{0}$-norm minimization, $l_{1}$-norm minimization is used to substitute $l_{0}$-norm in many practical cases. However, for image processing, recent research has proved that the nonlinear total variation (TV) minimization can preserve more image edges or more accurate boundaries of the recovered image than $l_{1}$-norm minimization due to the fact that most images are sparse in their gradients ${ }^{26}$. The concept of $T V$ minimization was introduced to compressive sensing field by Candes et al24. And was mathematically described as

$$
T V(x)=\sum_{i}\left\|(\nabla x)_{i}\right\|_{p}
$$

where $\nabla$ is a gradient operator. In the present work, we adopt a single step TV based minimization algorithm in all three-dimensions (3D) to reconstruct the pixel value $P \in \mathbb{R}^{n_{1} \times n_{2} \times n_{3}}$ in the $3 \mathrm{D}$-OCT image volumetric image. Then the Eq. (2) becomes

$$
\underset{x}{\operatorname{argmin}} \sum_{i, j, k}\left\|(\nabla(L x))_{i, j, k}\right\|_{1} \text { s.t. } A x=y
$$

Obviously, the Eq. (5) is an equality constrained problem, according to the numerical optimization theory, then Eq. (5) can be transformed into an unconstrained minimize to recover $\mathrm{x}$ :

$$
\underset{x}{\operatorname{argmin}}\left\{\sum_{i, j, k}\left\|(\nabla(L x))_{i, j, k}\right\|_{1}+\frac{\mu}{2}\|A x-y\|^{2}\right\}
$$

where the second term is a quadratic penalty approach that penalizing the constraint violations with a balancing parameter $\mu$. Hence, we can obtain the solution $x^{*}$ by solving the Eq. (6), and the original 3D OCT volumetric image has been perfectly reconstructed.

It is worth noting that in a Fourier domain OCT system (FD-OCT), the image is recovered by taking the inverse Fourier transform. Thus, the linear transformation operator $L$ in Eq. 5 need to be replaced by the Fourier transform operator $(F F T)$, which is defined as

$$
F F T(\omega)=\sum_{i} x \exp (-i \omega t)
$$

However, in a conventional i.e. time domain OCT (TD-OCT) system, the transformation operator $L$ can be neglected since no such a transformation need to be conducted.

\section{Results}

We simulated the proposed single step fully 3D CS OCT image sampling and reconstruction strategy by applying it onto a Fourier (or 
spectral) domain OCT volumetric data of a human retina, which was acquired by a commercial FD-OCT system (iVue OCT system, Optovue). The original 3D OCT volumetric image has a size of 128 $\times 128 \times 100$ volumetric pixels as shown in Fig. 4 . The simulation was implemented with MATLAB R2014a software package run on a desktop consisted of an Intel (R) Core (TM) i7 CPU (3.96 GHz), 32GB RAM, windows 10 64bit operation system. In order to have a more quantitative evaluation of the quality of the reconstructed $3 \mathrm{D}$ volumetric image, we calculated the peak signal-to-noise ratio (PSNR) of the recovered 3D volumetric image $R(i, j, k)$ to the original 3D volumetric image $I(i, j, k)$. PSNR is defined as

$$
P S N R=10 \log _{10} \text { peakval } / \text { MSE }
$$

where peakval is the max amplitude of the reconstructed 3D volumetric image, and MSE is the mean square error which can be estimated as

$$
M S E=\frac{1}{N} \sum_{i, j, k}[I(i, j, k)-R(i, j, k)]^{2}
$$

which $N=n_{1} \times n_{2} \times n_{3}$ is the total pixels of the $3 \mathrm{D}$ volumetric image.
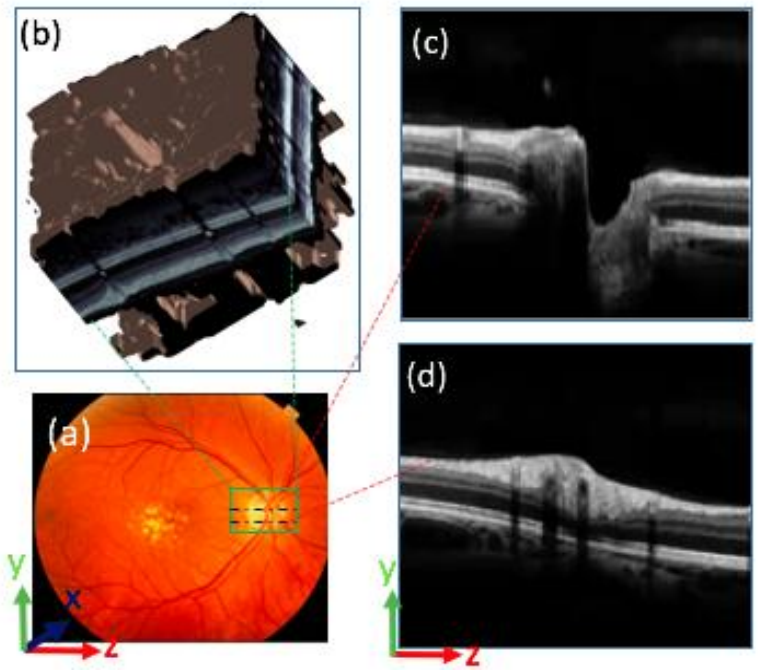

Fig. 4. The original volumetric image data set for single step fully 3D CS reconstruction was taken from a) the human eye $\mathrm{e}^{7}$ and the highlighted green dash rectangular is the region of OCT volume acquisition ${ }^{7}$, b) 3D visualization of the retina OCT volume image, c) and (d) are 2D of the center cross sections (B-scan) taken from the OCT volume data set (the two black dash lines in the region of picture a).

Generally, the measurement sampling rate, set to the ratio of the number of measurements to the length of the original signal, could have an important impact on the resolution of the recovered signal. To this end, a wide range of sampling rates from $10 \%$ to $60 \%$ was chosen for investigating the relation between the compressive sensing sampling rate and PNSR in our proposed approach as shown in Fig. 5. It is clear that the image PNSR increases with the sampling rate. More importantly, it shows that a $P S N R$ value near to or greater than $38 \mathrm{~dB}$ can be reached by using only $10 \%$ of the total 3D OCT volumetric image measurements. In other words, a lower sampling rate can be utilized to recover a 3D CS-OCT volumetric image without losing image quality. Naturally, the higher the PSNR value is, the better the quality of the recovered image is. This advantage of the new imaging technique would be most preferably in the field of life science. However, with the sampling rate increasing, the PSNR first increases at a higher rate until $40 \%$ sampling rate and then have a tendency to become flattened or saturated as shown in Fig. 5.
Moreover, in order to exhibit the efficiency of our proposed method, the PSNR of the majority of 3D CS reconstruction approaches based on 2D CS slices one by one of the same human eye volumetric image was also calculated as shown in Fig.5. Obviously, it has the similar tendency in the relation between the sampling rate and PSNR value, but nearly $8 \mathrm{~dB}$ lower PSNR than that of our proposed method.



Fig. 5. PSNR of the original image to the reconstructed of human retina versus the sampling rate.

In order to visualize the performance of our proposed approach, two reconstructed 3D volume images with a sampling rate of $10 \%$ and $30 \%$ respectively were illustrated in Fig. 6 . It shows that, compared with the original image, although the reconstructed image with $10 \%$ sampling rate is slightly noisy, the edge of the image is well preserved still resulting in a relatively good quality as shown in Fig. 6(b). Certainly, the image quality can be easily improved by increasing the sampling rate as stated above and the PSNR is dramatically increased by $10 \mathrm{~dB}$ with the sampling rate increased to $30 \%(P S N R=48.3)$ as shown in Fig. 6(c). To further explore internal details in the 3D reconstructed image, four B-scans slices at different locations from each of the original and two reconstructed images with different sampling rates were selected for comparison as shown in Fig. 7. As we stated before, these results demonstrated that the proposed technique is effective in 3D imaging and exhibited a good potential to reconstruct the 3D OCT volumetric image with a far lower sampling rate.
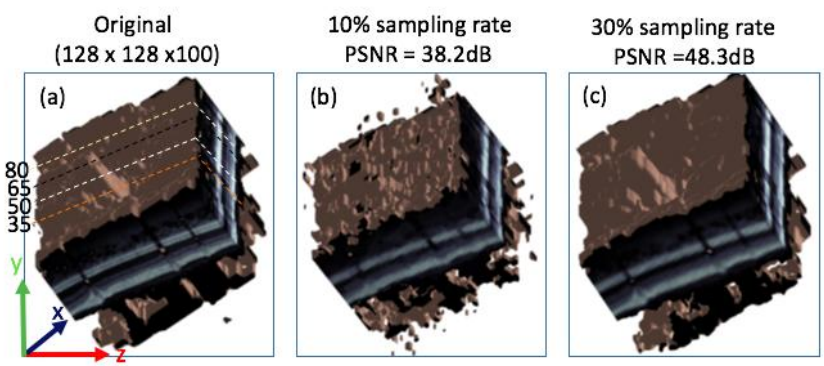

Fig. 6. Illustration of the human retina volumetric images of a) the original, and the reconstructed by fully single step 3D CS algorithm with a sampling rate of b) $10 \%(P S N R=38.2 \mathrm{~dB}), \mathrm{c}) 30 \%(P S N R=48.3$ 
$\mathrm{dB})$.

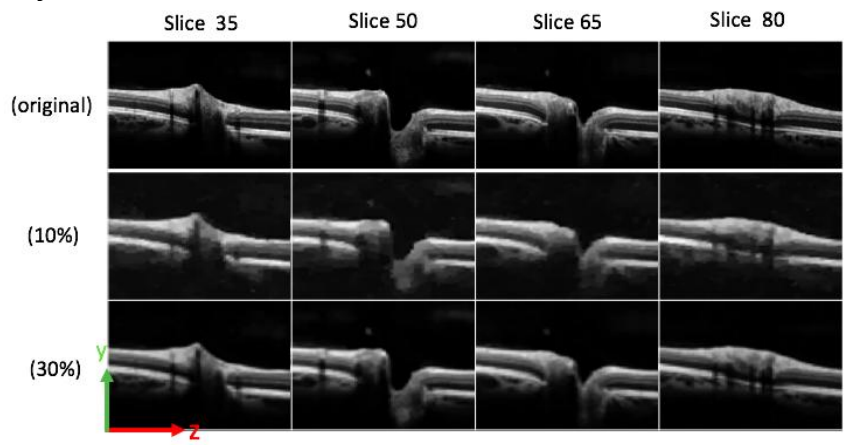

Fig. 7. Four slices are chosen from the single step fully 3D CS reconstruction of the human retina OCT volumetric image $(128 \mathrm{x} 128 \mathrm{x}$ 100 ), and each slice has $128 \times 128$ pixels. The top, the middle and bottom rows show the detail of the four B-scan slices that were extracted from the original, $10 \%$ and $30 \%$ sampling rate respectively; and the positions of these selected slices in the volumetric OCT image with different dash line colors are also showed in Fig. 6(a).

\section{Conclusions}

In this paper, we have proposed and demonstrated a novel single step fully 3D CS-OCT volumetric image reconstruction approach, in which CS is implemented in all three dimensions. The simulations show that the proposed approach can be used to reconstruct the 3D OCT volumetric images with high resolution but far fewer measurements (less than 10\%). This novel method can be also applied to some other medical image systems, such as magnetic resonance imaging (MRI) and Computer Tomography (CT).

Acknowledgment. We thank Guoqing $\mathrm{Hu}$, Jie Chen for their valuable discussions and help, and we also greatly appreciate the ophthalmology of Ningxiang traditional Chinese medicine hospital for providing the original OCT data. This work was supported by National Science Foundation of China (51328601), National Instrumentation Program (2011YQ0301240502) and the Aviation Science Foundation of China (2011ZD51049).

\section{References}

1. Baran, U., Qin, W., Qi, X., Kalkan, G. \& Wang, R. K. OCT-based label-free in vivo lymphangiography within human skin and areola. Scientific reports 6, 21122 (2016).

2. Jung, Y., Klein, O. J., Wang, H. \& Evans, C. L. Longitudinal, label-free, quantitative tracking of cell death and viability in a 3D tumor model with OCT. Scientific reports 6, 27017 (2016).

3. Sun, Z. et al. An automated framework for 3D serous pigment epithelium detachment segmentation in SD-OCT images. Scientific reports 6, 21739 (2016).

4. Chang, F. Y. et al. Optical coherence tomography-guided laser microsurgery for blood coagulation with continuous-wave laser diode. Scientific reports 5, 16739 (2015).

5. Kennedy, K. M. et al. Quantitative micro-elastography: imaging of tissue elasticity using compression optical coherence elastography. Scientific reports 5, 15538 (2015).

6. Wierwille, J. et al. In vivo, label-free, three-dimensional quantitative imaging of kidney microcirculation using Doppler optical coherence tomography. Lab Invest 91, 1596-1604 (2011).

7. Zawadzki, R. J. et al. 3D OCT imaging in clinical settings: toward quantitative measurements of retinal structures. Proceedings of SPIE The International Society for Optical Engineering 6138 (2006).
8. Wieser, W. et al. High definition live 3D-OCT in vivo: design and evaluation of a 4D OCT engine with $1 \mathrm{GVoxel} / \mathrm{s}$. Biomedical optics express 5, 2963-2977 (2014).

9. Liu, T. \& Wu, K. High-speed optical coherence tomography based on line scanning. Proceedings of SPIE - The International Society for Optical Engineering (2002).

10. Kim, J.-h., Han, J.-H. \& Jeong, J. Adaptive optimization of reference intensity for optical coherence imaging using galvanometric mirror tilting method. Optics Communications 351, 57-62(2015).

11. Wang, R. K. \& Ma, Z. A practical approach to eliminate autocorrelation artefacts for volume-rate spectral domain optical coherence tomography. Physics in medicine and biology 51, 3231-3239(2006).

12. Hillmann, D. et al. Common approach for compensation of axial motion artifacts in swept-source OCT and dispersion in Fourier-domain OCT. Opt. Express 20, 6761-6776 (2012).

13. Fang, L. et al. Sparsity based denoising of spectral domain optical coherence tomography images. Biomedical optics express 3, 927-942 (2012).

14. Xu, D., Huang, Y. \& Kang, J. U. Real-time compressive sensing spectral domain optical coherence tomography. Optics Letters 39, 76-79 (2014).

15. Lebed, E., Mackenzie, P. J., Sarunic, M. V. \& Beg, F. M. Rapid Volumetric OCT Image Acquisition Using Compressive Sampling. Opt. Express 18, 21003-21012 (2010)

16. Beg, M. F., Lebed, E. \& Sarunic, M. V. System and method for rapid OCT image acquisition using compressive sampling. (2015).

17. Candes, E. J. \& Wakin, M. B. An Introduction To Compressive Sampling. IEEE Signal Processing Magazine 25, 21-30 (2008).

18. Soldevila, F., Salvador-Balaguer, E., Clemente, P., Tajahuerce, E. \& Lancis, J. High-resolution adaptive imaging with a single photodiode. Scientific reports 5, 14300 (2015).

19. Yu, W. K. et al. Complementary compressive imaging for the telescopic system. Scientific reports 4, 5834 (2014).

20. Huynh, N. et al. Single-pixel optical camera for video rate ultrasonic imaging. Optica 3 (2016).

21. Lebed, E., Lee, S., Sarunic, M. V. \& Beg, M. F. Rapid radial optical coherence tomography image acquisition. Journal of Biomedical Optics 18, 537-543 (2013).

22. Xu, D., Huang, Y. \& Kang, J. U. Volumetric (3D) compressive sensing spectral domain optical coherence tomography. Biomedical optics express 5, 3921-3934 (2014).

23. Donoho, D. L. Compressed Sensing. IEEE Transactions on Information Theory 52, 1289-1306 (2006).

24. Candes, E. J., Romberg, J. \& Tao, T. Robust uncertainty principles: exact signal reconstruction from highly incomplete frequency information. IEEE Transactions on Information Theory 52, 489-509 (2006).

25. Cohen, A., Dahmen, W. \& Devore, R. Compressed sensing and best kterm approximation. Journal of the American Mathematical Society 22, 211-231 (2009).

26. Yip, T. C. S. E. F. P. A. Recent Developments In Total Variation Image Restoration. Mathematical Models of Computer Vision 5 (2005). 